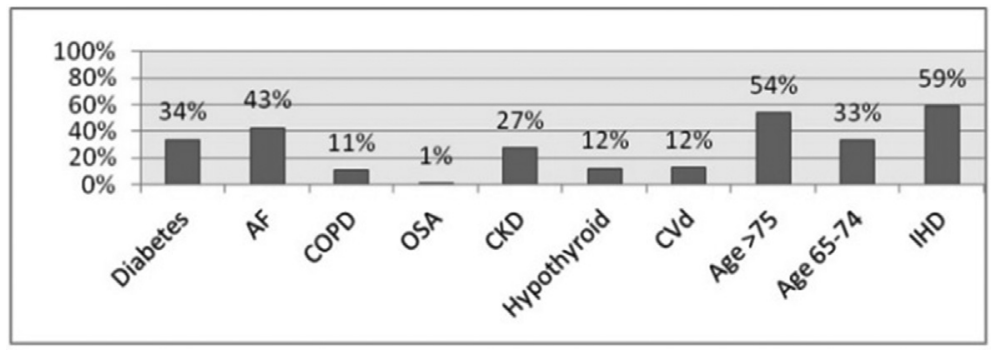

Abstract 19 Figure 1 Modified Frailty Index Clinical parameters and prevalence in our study population (Potential for a maximum and minimum modified frailty index of 11 and 0 )

surprisingly small in medium term follow up. This implies that clinician guided selection criteria is an appropriate method of selecting patients who may not derive additional benefit from a defibrillator.

\section{MODIFIED FRAILITY INDEX AS PREDICTOR OF OUTCOME FOR PATIENTS IMPLANTED WITH CARDIAC RESYNCHRONISATION THERAPY}

"Shveta Monga, Dominic Haigh, David Royan, Chitsa Seyani, Richard Francis, Paul Foley, Badrinath Chandrasekaran. 'Great Western hospital NHS trust

\subsection{6/heartjnl-2017-311726.19}

Introduction Frailty is a multidimensional syndrome, contributing significantly to the morbidity and mortality of elderly patients. With an increase in average life expectancy, there are a growing number of frail individuals with multiple co-morbidities and cardiac failure. Although multiple studies have shown that in selected patients cardiac resynchronisation therapy (CRT) improves morbidity and mortality, the role of frailty has not been rigorously evaluated in these individuals. There are no current risk scores for frailty to help refine the predicted impact of CRT on survival in elderly patients.

Study aim and basic methods The focus of our study was to develop and review the role of an easy-to -use frailty index as a predictor of outcome in patients being considered for CRT and suggest ways in which frailty assessment could be incorporated into clinical practice.

This was a retrospective study of 265 patients implanted with CRT from 2011 to 2015. Data was collected from

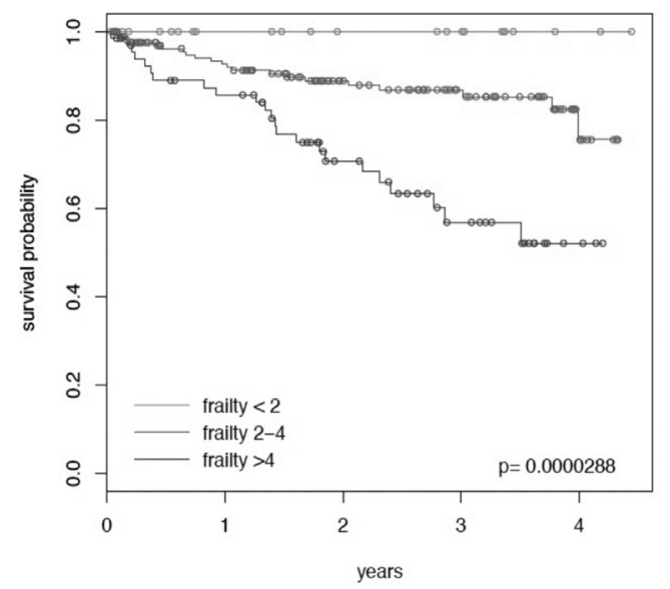

patient electronic records and follow-up visits. The median follow-up duration was $2.2(1.2-3.3)$ years.

A modified frailty index was formulated using the Canadian Study of Health and Ageing. The modified frailty index was based on 9 of the potential 70 Canadian Study of Health and Ageing clinical deficits. These variables were chosen based on the likelihood of being recorded in the electronic records bearing in mind the practical future application of the frailty index in a clinical setting. Each deficit was given 1 point except age $>75$ years (given 2 points). The clinical parameters used and their prevalence in our study population is shown in figure 1 .

Data were analysed using SPSS and Microsoft excel using Chi square test, survival analysis and ROC analysis.

Results 1. In patients implanted with CRT, mortality was found to be higher in patients with a greater frailty index. ( $\mathrm{p}$ value 0.00003 ) (Image 1)

2. Mean modified frailty index in those who died was higher (4.4; SD 1.5) than in those who lived (3.1; SD 1.5)

3 . In ROC analysis, frailty index $>2$ was a significant predictor of mortality in our subgroup of patients (sensitivity 97.7\%, AUC 0.73, 95\% CI=0.14-0.32, p<0.0001) (Image 2)

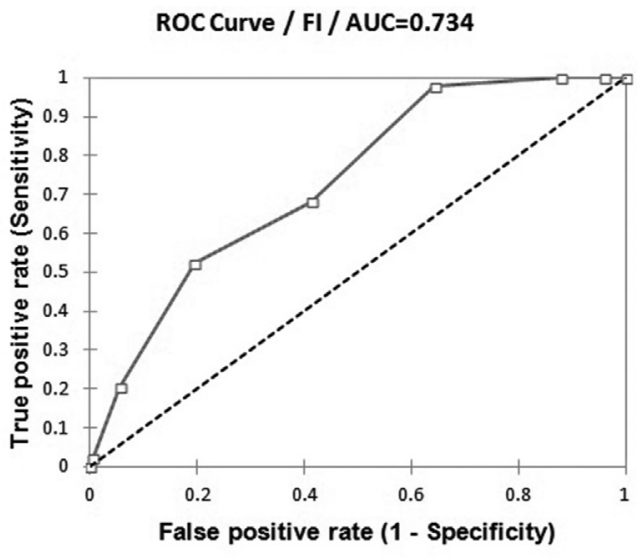

Abstract 19 Figure 3

Conclusion In patients implanted with CRT mortality was found to be significantly greater in more frail patients, represented by a higher modified frailty index. Therefore the modified frailty index may be a useful tool in predicting mortality, helping to guide patient and family expectations when considering device therapy. 